\title{
Formalin-fixed, paraffin-embedded (FFPE) tissue epigenomics using Infinium HumanMethylation450 BeadChip assays
}

\author{
Tim C de Ruijter ${ }^{1,2}$, Joep PJ de Hoon ${ }^{1,2}$, Jeroen Slaats ${ }^{1,2}$, Bart de Vries ${ }^{2,3}$, Marjolein JFW Janssen ${ }^{4}$, Tom van Wezel ${ }^{5}$, \\ Maureen JB Aarts ${ }^{1,2}$, Manon van Engeland ${ }^{2,3}$, Vivianne CG Tjan-Heijnen ${ }^{1,2}$, Leander Van Neste ${ }^{2,3,7}$ and \\ Jürgen Veeck ${ }^{1,2,6,7}$
}

Current genome-wide methods to detect DNA-methylation in healthy and diseased tissue require high-quality DNA from fresh-frozen (FF) samples. However, well-annotated clinical samples are mostly available as formalin-fixed, paraffinembedded (FFPE) tissues containing poor-quality DNA. To overcome this limitation, we here aimed to evaluate a DNA restoration protocol for usage with the genome-wide Infinium HumanMethylation450 BeadChip assay (HM-450K). Sixty-six DNA samples from normal colon $(n=9)$ and breast cancer $(n=11)$ were interrogated separately using HM-450K. Analyses included matched FF/FFPE samples and technical duplicates. FFPE DNA was processed with (FFPEr) or without a DNA restoration protocol (Illumina). Differentially methylated genes were finally validated in 24 additional FFPE tissues using nested methylation-specific PCR (MSP). In summary, $\beta$-values correlation between FFPEr duplicates was high $(\rho=0.9927$ (s.d. \pm 0.0015$)$ ). Matched FF/FFPEr correlation was also high $(\rho=0.9590$ (s.d. \pm 0.0184$)$ ) compared with matched FF/FFPE $(\rho=0.8051$ (s.d. \pm 0.1028$)$. Probe detection rate in FFPEr samples $(98.37 \%$, s.d. \pm 0.66$)$ was comparable to FF samples $(99.98 \%$, s.d. \pm 0.019$)$ and substantially lower in FFPE samples $(82.31 \%$, s.d. \pm 18.65$)$. Assay robustness was not decreased by sample archival age up to 10 years. We could also demonstrate no decrease in assay robustness when using $100 \mathrm{ng}$ of DNA input only. Four out of the five selected differentially methylated genes could be validated by MSP. The gene failing validation by PCR showed high variation of $\mathrm{CpG} \beta$-values in primer-binding sites. In conclusion, by using the FFPE DNA restoration protocol, $\mathrm{HM}-450 \mathrm{~K}$ assays provide robust, accurate and reproducible results with FFPE tissue-derived DNA, which are comparable to those obtained with FF tissue. Most importantly, differentially methylated genes can be validated using more sensitive techniques, such as nested MSP, altogether providing an epigenomics platform for molecular pathological epidemiology research on archived samples with limited tissue amount.

Laboratory Investigation (2015) 95, 833-842; doi:10.1038/labinvest.2015.53; published online 13 April 2015

Epigenomic changes are recognised as important factors in tumour initiation, growth and progression. Global and local DNA-methylation patterns are frequently altered in cancer cells, resulting in genomic instability and diminished or elevated expression of tumour-suppressor genes or oncogenes, respectively, driving malignant transformation, growth promotion and metastasis (reviewed in Jones and Baylin ${ }^{1}$ and Petronis $^{2}$ ). In the clinical setting, cancer-specific DNA-methylation changes are increasingly evaluated as biomarkers for early detection, staging or patient prognosis.
In addition, a role for DNA-methylation changes in mediating either cancer drug resistance or drug sensitivity has been hypothesised and verified. ${ }^{3-6}$ Therefore, the in-depth study of the cancer DNA-methylome holds promise to provide important clues as to which genes and biological networks are affected at tumour initiation and progression. Furthermore, it will provide clues as to which genes are epigenetically affected during escape from therapeutic cytotoxicity.

Methylation of cytosine residues within cytosine-phosphate-guanine $(\mathrm{CpG})$ dinucleotides is a stable chemical

'Division of Medical Oncology, Maastricht University Medical Center, Maastricht, The Netherlands; ${ }^{2}$ GROW—School for Oncology and Developmental Biology, Maastricht University Medical Centre, Maastricht, The Netherlands; ${ }^{3}$ Department of Pathology, Maastricht University Medical Centre, Maastricht, The Netherlands; ${ }^{4}$ ServiceXS, Leiden, The Netherlands; ${ }^{5}$ Department of Pathology, Leiden University Medical Centre, Leiden, The Netherlands and ${ }^{6}$ Institute of Pathology, Medical Faculty, RWTH Aachen University Hospital, Aachen, Germany

Correspondence: Dr J Veeck, PhD, Institute of Pathology, RWTH Aachen University Hospital, Pauwelsstr. 30, Aachen 52074, Germany.

E-mail: juergen.veeck@rwth-aachen.de

7 These authors contributed equally to this work.

Received 12 January 2015; revised 6 March 2015; accepted 7 March 2015 
modification of the genomic DNA. CpG methylation can be analysed after nucleic acid isolation from various cellular and cell-free sources. ${ }^{7}$ Several techniques to analyse CpG methylation have been developed, encompassing locus-specific methods that interrogate single to few genes as well as genome-wide and whole genome methods capturing the epigenomic landscape in a high-throughput manner. For biomarker discovery studies, mostly genome-wide methods are applied as these enable comparisons of large parts of the DNA-methylome between different sample groups. Genomewide discovery analyses provide at best candidate loci that still need to be validated with locus-specific assays, such as methylation-specific PCR (MSP) or bisulphite sequencing methods. Not only are these techniques considered superior in determining methylation status, they are also more cost and time efficient for the analysis of tissue biomarkers as diagnostic tools. ${ }^{8}$

Although bisulphite sequencing represents the gold standard for finding differentially methylated DNA sequences at single-nucleotide resolution, currently both whole genome and genome-wide bisulphite sequencing techniques are considered too costly for applications with larger sample numbers. ${ }^{9,10}$ As an alternative, the BeadArray technique, designed by Illumina, has been widely used to interrogate DNA-methylation in a genome-wide manner, although with less coverage than genome-wide bisulphite sequencing. The first version of this assay, the Infinium HumanMethylation27 BeadChip assay (HM-27K), ${ }^{11}$ has been recently outdated by its successor platform, the Infinium HumanMethylation450 BeadChip assay (HM-450K). ${ }^{12}$ In comparison, this platform is lower in cost than whole genome bisulphite sequencing, but similar to all array-based platforms it is biased towards the design of its probe locations. Despite this, it can be used to analyse the methylation status of $>485000$ known CpG sites throughout the human genome, covering $96 \%$ of all $\mathrm{CpG}$ islands and promoters of $99 \%$ of known RefSeq genes. ${ }^{12}$ This technique has been extensively validated by several studies, both in comparison to its predecessor (HM-27K) and the gold standard, ie, bisulphite sequencing. ${ }^{12-14}$

Similar to other array-based technologies that include a whole genome amplification step, HM-450K was primarily designed for high-quality DNA, which is obtained from freshfrozen (FF) tissue, blood and blood-borne cells or cell lines. With respect to cancer, however, formalin-fixed, paraffinembedded (FFPE) tissue is more widely accessible. Hospitals all over the world manage archives containing vast amounts of FFPE tumour tissue samples, which could yield clinically valuable epigenomic information. Importantly, FFPE tissuederived DNA is generally of poor integrity as characterised by DNA-protein crosslinks and nucleic acid fragmentation, ${ }^{15}$ rendering this source of DNA less compatible with HM-450K. Recently, optimised protocols have been suggested aiming to improve FFPE DNA quality in order to achieve robust array results. The 'ligation method', based on ligating fragmented FFPE DNA using a ligase reaction before bisulphite conversion, ${ }^{16}$ has been used in combination with $\mathrm{HM}-27 \mathrm{~K}$ assays, but the reproducibility of this technique has been questioned as it may have a high false prediction rate in selecting differentially methylated probes. ${ }^{16-18}$ An alternative 'restoration method' improves FFPE DNA by chemical correction of base pair lesions, DNA crosslinks and base modifications after bisulphite conversion but has only been used in few array-based studies so far; ${ }^{19-21}$ hence, little evidence supporting the feasibility of this technology has been published.

Here we evaluate the use of FFPE-derived DNA after restoration for HM-450K assays using the Infinium FFPE DNA Restoration solution (Illumina). We provide a proof-ofprinciple study showing that genome-wide methylation analysis on aged, archived FFPE tumour tissues is feasible after improvement of DNA quality.

\section{MATERIALS AND METHODS \\ Sample Characteristics}

The data used for this study were derived from two separate subsets using HM-450K analysis. The first subset consisted of normal colonic mucosa $(n=9)$ samples at different input amounts. Based on findings of the first subset, a second subset consisting of invasive breast cancer samples $(n=11)$ was processed. Samples were obtained from pathological archives of Leiden University Medical Centre (normal colon) and Maastricht University Medical Centre (breast cancer).

In addition to FFPE samples, for five of the nine colon samples matched FF tissue was available. FF samples have been collected by snap-freezing in liquid nitrogen immediately after surgery. Median archival age of normal colon samples was 5 years (range 3-25 years).

Breast cancer samples were selected from the transDATA biospecimen registry, a currently conducted multi-centre array study on hormone receptor (HR)-positive, invasive breast cancer. Median archival age of breast cancer samples was 8 years (range $8-10$ years).

A third cohort of primary FFPE tissue samples, aimed for validation purposes, consisted of 12 normal colonic mucosa tissues (median archival age 17 years (range 16-18 years) and 12 HR-positive invasive breast tumour tissues (median archival age 9 years (range 7-10 years)) obtained from the Department of Pathology, Maastricht University Medical Center. All samples used in this study were anonymised, according to the Dutch code of conduct for responsible use of human tissue. ${ }^{22,23}$

\section{Sample Preparation and DNA Extraction}

Consecutive sections from FF normal colonic mucosa samples were used after expert review of haematoxylin/ eosin-stained sections confirmed both the absence of tumour cells as well as an epithelial cellularity of about $70 \%$ in each sample. DNA from FF colon samples was extracted using the Nucleospin Tissue Kit (Macherey-Nagel, Düren, Germany) according to the manufacturer's instructions. To assure a high 
percentage of epithelial cells, FFPE tissue sections were marked by an expert pathologist and macrodissected from the slides aiming at an epithelial cell content of at least $70 \%$. Normal colon FFPE tissue DNA was extracted from eight 10$\mu \mathrm{m}$ slides using the Nucleospin FFPE Tissue Kit (MachereyNagel) according to the manufacturer's instructions. Breast tumour FFPE tissue DNA was extracted from eight $10-\mu \mathrm{m}$ slides, using the Maxwell FFPE CSC Automated DNA Extraction Kit (Promega Corporation, Madison, USA) according to the manufacturer's instructions. DNA concentrations were measured using the PicoGreen assay (Life Technologies, Bleiswijk, The Netherlands).

\section{DNA Restoration Procedure}

DNA restoration was performed using the Illumina HD FFPE Restoration Kit (Illumina, San Diego, USA) according to the manufacturer's instructions. Intermediate DNA purifications were performed using the Zymo DNA Clean and Concentrator-5 Kit (Zymo Research, Irvine, USA).

\section{Infinium HM-450K}

In total, $n=66 \mathrm{HM}-450 \mathrm{~K}$ assays were conducted in this study. Normal colon and breast cancer samples were assayed in different batches. In short, DNA samples were first examined for fragmentation by electrophoresis on $2 \%$ agarose gels. All FF samples showed high integrity with a single band $>10 \mathrm{~kb}$. All FFPE samples $<10$ years of age showed a smear around $1-2 \mathrm{~kb}$. The older FFPE samples showed increased fragmentation and lower fragment sizes. Samples were bisulphiteconverted using the EZ DNA Methylation Gold Kit (Zymo Research) according to the manufacturer's instructions. To verify bisulphite conversion quality, quantitative PCR reactions and melting curve analyses were performed as previously described. ${ }^{24}$ The Infinium protocol was directly applied to FF samples, while colon FFPE samples were analysed by Infinium both with and without DNA restoration and breast cancer FFPE samples with DNA restoration. Bisulphite-treated samples underwent whole genome amplification, followed by fragmentation and hybridisation to the Infinium HM-450K, as validated by Sandoval et al. ${ }^{12}$ and following the Infinium HD FFPE protocol provided by Illumina. Varying amounts of DNA input at bisulphite conversion were evaluated for colon samples, ranging from $62.5-500 \mathrm{ng}$ of DNA. Based on the results of varying input amounts (Supplementary Figure S1), a constant DNA input of $100 \mathrm{ng}$ was used for breast cancer samples. A detailed description of procedures and input characteristics for all samples as well as age at the time of DNA isolation are described in Supplementary Figures S2a-c.

\section{Data Processing and Statistical Analysis}

Chips were scanned using the iScan system (Illumina). iScan data were extracted to the GenomeStudio software v2011.1 using default analysis settings. No normalisation was performed on sample data. The methylation status for each probe was displayed as a $\beta$-value that ranged between 0 and 1 , where values close to 1 represent high levels of methylation and those close to 0 represent low levels of methylation. A detection $P$-value based on the fluorescence intensity was calculated for all probes. Probes that did not yield a significant intensity over background ( $P$-value $\geq 0.01)$ were excluded from the analysis. All correlation analyses were performed using Spearman's correlation test. HM-450K data for each analysed sample has been deposited in the Gene Expression Omnibus (GEO) data repository and can be accessed by the study identifier GSE66555.

\section{Gene and Area of Interest Selection}

Differentially methylated $\mathrm{CpG}$ sites were selected by two single-sided $t$-tests. Probes were considered to be differentially methylated at a $P$-value $<0.001$. Of these statistically significant differentially methylated probes, those with a $\beta$ value $<0.2$ in the low-methylation group were selected and mapped back to the human genome. Areas of interest were defined as DNA sequences of a maximum of 200 base pairs (bp) in length within a region from 2 kilobases $(\mathrm{kb})$ upstream to $500 \mathrm{bp}$ downstream relative to the transcription start site, containing three or more differentially methylated probes.

\section{MSP Validation}

MSP primers were designed for all the selected areas of interest. Validation samples were bisulphite-converted using the EpiTect Bisulfite Kit (Qiagen, Hilden, Germany). Nested multiplex MSP was performed as previously described. ${ }^{25-27}$ In short, a non-discriminatory (multiplexed) PCR reaction was performed with $200 \mathrm{ng}$ of bisulphite-treated genomic DNA input using methylation-independent primers flanking the area of interest. Four microlitres of non-discriminatory PCR product (diluted 1:1000) was then used for discriminatory MSP reactions using primers specific for methylated or unmethylated DNA. Methylation status was assessed using $3 \%$ gel electrophoresis of the MSP product. Primers and reaction specifications are listed in Supplementary Table S1.

\section{RESULTS}

A total of $n=44 \mathrm{HM}-450 \mathrm{~K}$ analyses were performed with colon samples, of which one restored FFPE (FFPEr) sample failed internal quality control and was excluded from further analysis. The 11 breast cancer DNA samples were restored and analysed as technical duplicates on the HM-450K array $(n=22)$. Internal controls of the HM-450K analyses were well within limits for all breast cancer samples.

\section{Correlation Analyses of Duplicate Samples and Matched Sample Types}

In order to determine the robustness of the HM-450K assay when applied to FFPE-derived DNA, we first assessed assay reproducibility and calculated the Spearman's correlation coefficient (rho, $\rho$ ) of the FF, FFPE and FFPEr technical duplicates available for colon sample \#6. The $\rho$-value of the 
FF DNA duplicates was the highest (0.9978) and the $\rho$-value of FFPEr DNA duplicates was comparable (0.9891), in contrast to the $\rho$-value for unrestored FFPE DNA duplicates (0.8960) (Figure 1a). Correlation between sample \#6 technical duplicates reflected the inter-duplicate correlation of other FFPEr samples as well. Mean $\rho$-value of FFPEr technical duplicates of all colon and breast cancer samples was 0.9927 (s.d. \pm 0.0015 ).

HM-450K includes 65 probes measuring single-nucleotide polymorphisms (SNPs), which can be used for fingerprinting purposes, ie, confirming the origin or identity of samples. We used these probes to compare concordance between matched FF, FFPE and FFPEr biological duplicates (samples \#5 to \#9). Concordance of SNP status between matched FF and FFPEr samples $(\rho=0.986)$ was highly similar to the concordance of the FF duplicates $(\rho=0.998)$, as shown for colon sample \#6 (Figure $1 \mathrm{~b}$ ), indicating indeed similar SNP profiles between matched FF and FFPEr-DNA samples. In contrast, concordance of SNP status between matched FF and FFPE colon samples was low, achieving a $\rho$-value of only 0.788 .

Next, we calculated $\rho$-values between matched FF- and FFPE-derived methylation results, both from restored and unrestored FFPE colon DNA (Figure 1c). With a mean $\rho$-value of 0.9590 (s.d. \pm 0.0184 ), correlation between all five matched FF- and FFPEr-derived results (samples \#5 to \#9) was remarkably higher compared with the correlation between FFPE and FF results, which was only 0.8051 (s.d. \pm 0.1028 ). Density correlation plots of all technical and biological duplicates are depicted in Supplementary Figures S2d-f.

\section{Fluorescence Intensity and Probe Detection Rate Between Different Sample Types}

To further evaluate the robustness of the results from FFPEr tissue DNA, we analysed fluorescence intensity of the obtained probe signals. Combined intensity of the red and green fluorescence signals is an indicator of DNA yield, with a higher yield typically resulting in higher assay robustness. FF samples yielded a combined fluorescence intensity of 14187 (s.d. \pm 1089 ), compared with 9434 (s.d. \pm 1518 ) in FFPEr samples (Figure 2a). In order to investigate whether this decreased intensity in FFPEr could introduce bias in methylation readout, we compared the mean $\beta$-values of all probes for the five matched FF, FFPE and FFPEr colon samples. There was a significant difference in mean $\beta$-value between FF and FFPE-derived results, suggesting a bias towards unmethylated results in FFPE-derived data. However, this difference was no longer present when restored FFPEderived results were compared with FF results (Figure $2 \mathrm{~b}$ ).

Probe detection rate is another important parameter depending on fluorescence intensity. This is defined as the percentage of the 485550 probes for each sample that yields a signal discernible from background with a $P$-value $<0.01$. Thus, it represents the number of detectable CpG sites for which a methylation status can be measured. We analysed the effect of decreased fluorescence intensity on probe detection rate of the HM-450K assay when using FF, FFPE or FFPEr-derived DNA. Overall, in the FF colon samples $99.98 \%$ (s.d. \pm 0.019 ) of probes could be detected. In the FFPE colon samples, the mean probe detection rate was much less favourable $(82.31 \%)$ (s.d. \pm 18.65$)$. However, after DNA restoration the probe detection rate increased to 98.37\% (s.d. \pm 0.66 ) (Figure 2c). For all the abovementioned analyses, biological duplicate colon samples $\# 5$ to $\# 9$ were used.

\section{Effect of Sample Age on Assay Reproducibility}

In order to verify whether sample archival age affected assay robustness, we analysed the correlation of technical duplicate results derived from tissue blocks of varying age. Samples included in this study varied in age between 3 and 25 years. As input analyses showed a decrease in assay robustness when input amounts $<100 \mathrm{ng}$ were used (see Supplementary Figure S1), we only included duplicates of input amounts $\geq 100 \mathrm{ng}$. Using this restriction, 13 samples remained varying in archival age between 3 and 10 years and two samples of 25 years. Because no statistically sound conclusion could be made based on only two 25 -year-old samples, only 3-10-year-old samples were included for this analysis. Euclidean distances of these duplicates are shown in Figure 3. The slope of linear regression was not significant when comparing Euclidean distance and age of the sample, indicating that within the tested age range samples yield highly reproducible results.

\section{Unsupervised Hierarchical Clustering Analysis}

Abovementioned results indicate that the HM-450K assay can be robustly and reproducibly employed with restored FFPE tissue-derived DNA. To further test this hypothesis, we performed an unsupervised hierarchical clustering of all breast tumour duplicates and colon matched sample-derived results. This analysis showed correct clustering of all FF samples to their matched FFPEr counterparts, in contrast to FFPE unrestored samples, which generally did not cluster with their FF or FFPEr counterparts. Likewise, all breast cancer FFPEr technical duplicates showed high similarity (Figure 4).

\section{Impact of FFPEr-derived DNA on HM-450K Reproducibility}

Even though correlation between FFPEr duplicates applied to $\mathrm{HM}-450 \mathrm{~K}$ assay proved to be high, this correlation is lower when compared with FF duplicates. In order to visualise the impact of this slight decrease, we calculated the percentage of probes that indicated discordant $\beta$-values between technical duplicates and matched FF/FFPEr samples at different thresholds (Figure 5). For example, when a threshold for CG probe discordance is set at an absolute $\beta$-value difference of $0.1,0.6 \%$ of all probes showed discordant readout in the FF technical duplicate, compared with a considerable $30.8 \%$ in the FFPE duplicate, but only $5.7 \%$ in the FFPEr technical 
a
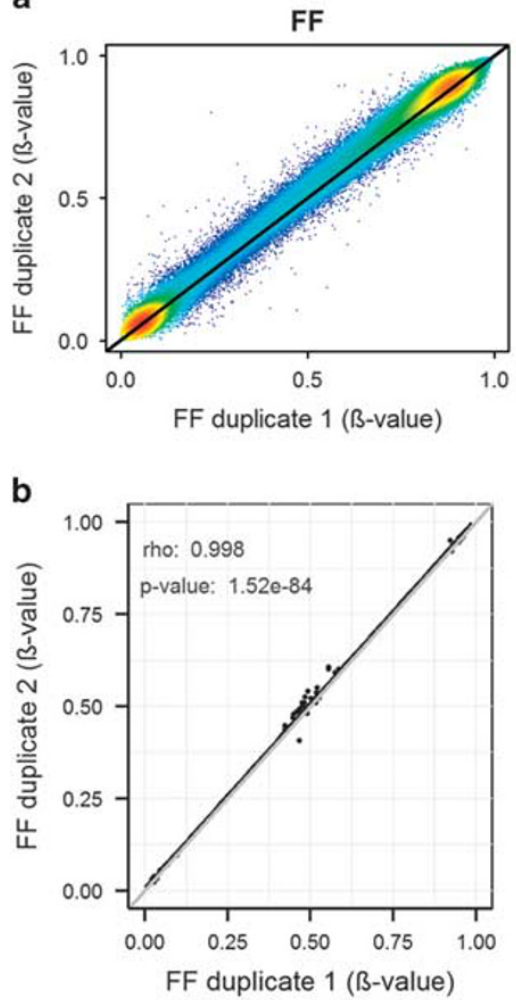

FFPE
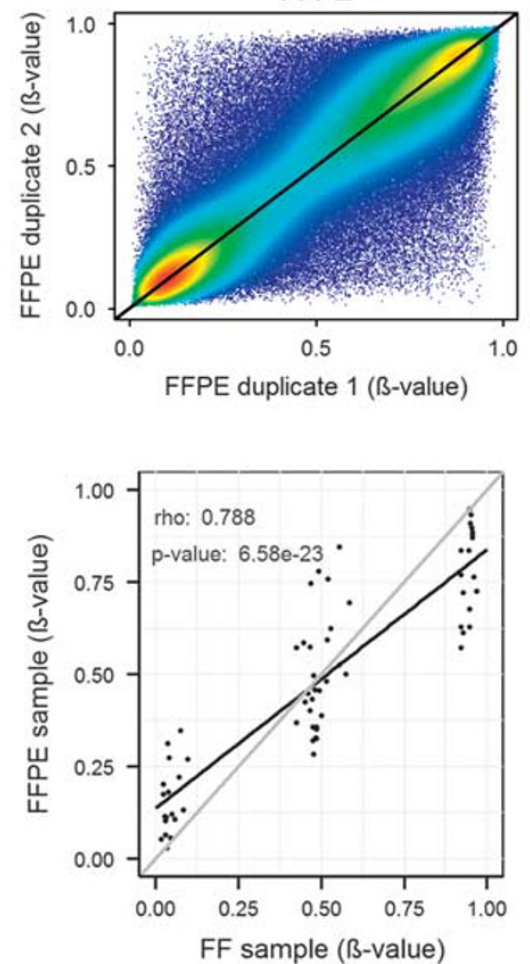

FFPEr
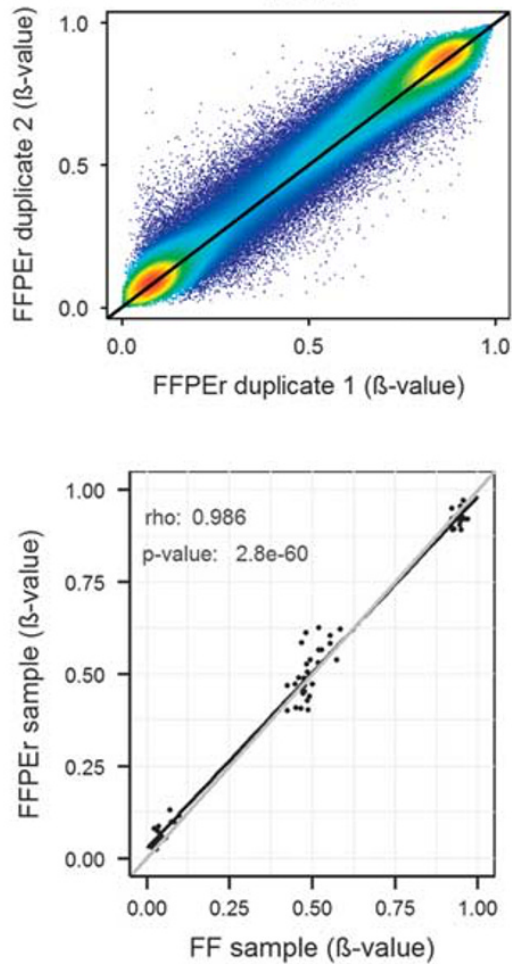

C

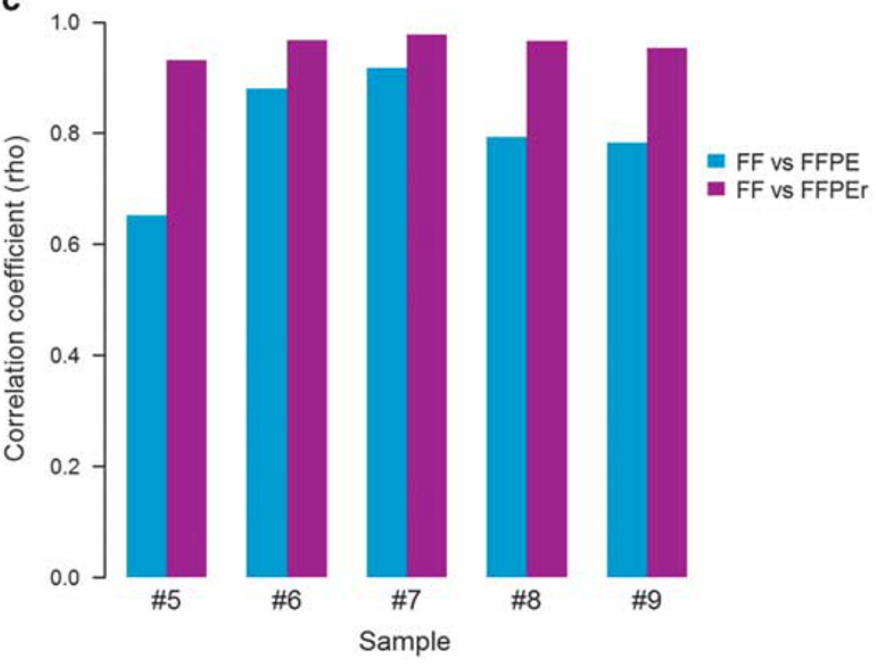

Figure $1 \mathrm{HM}-450 \mathrm{~K}$ assay robustness and reproducibility with DNA from FF tissue, FFPE tissue and restored FFPE tissue DNA. (a) Correlation analysis of technical duplicates of FF DNA, FFPE DNA and FFPEr DNA on HM-450K assays. Density correlation plots depict the correlation of normal colon sample \#6 (100 ng DNA input) between duplicate measurements of the same DNA after different treatments. Although correlation of untreated FFPE tissue DNA is poor $(\rho=0.8960)$ (middle plot), as compared with correlation of FF tissue DNA ( $\rho=0.9978)$ (left-hand side plot), it is substantially increased after treating FFPE tissue DNA according to the DNA restoration protocol $(\rho=0.9891)$ (right-hand side plot). Colours indicate point density and range from red (high point density) to blue (low point density). Density correlation plots for all technical and biological duplicates are shown in Supplementary Figures S2d-f. (b) Fingerprinting analysis of colon sample \#6 portraying high identity between gold standard FF duplicate results (left-hand side plot) while the SNP correlation decreases when comparing untreated FFPE tissue DNA to FF tissue DNA (middle plot) and increased to near gold standard levels after restoration (right-hand side plot). (c) Correlation between matched FF and FFPE colon samples compared with correlation between matched FF and FFPEr samples shows a clear increase in correlation in restored samples compared with unrestored samples.

duplicate (sample \#6). At a threshold of 0.2 , both FF- and FFPEr-derived results show concordance between technical duplicates in $>99 \%$ of the probes, whereas the FFPE-derived duplicates show discordant results in $10.9 \%$ of all probes (Figure 5a). As with the correlation analyses, matched samples from different origins, ie, FF, FFPE or FFPEr (samples \#5 to 

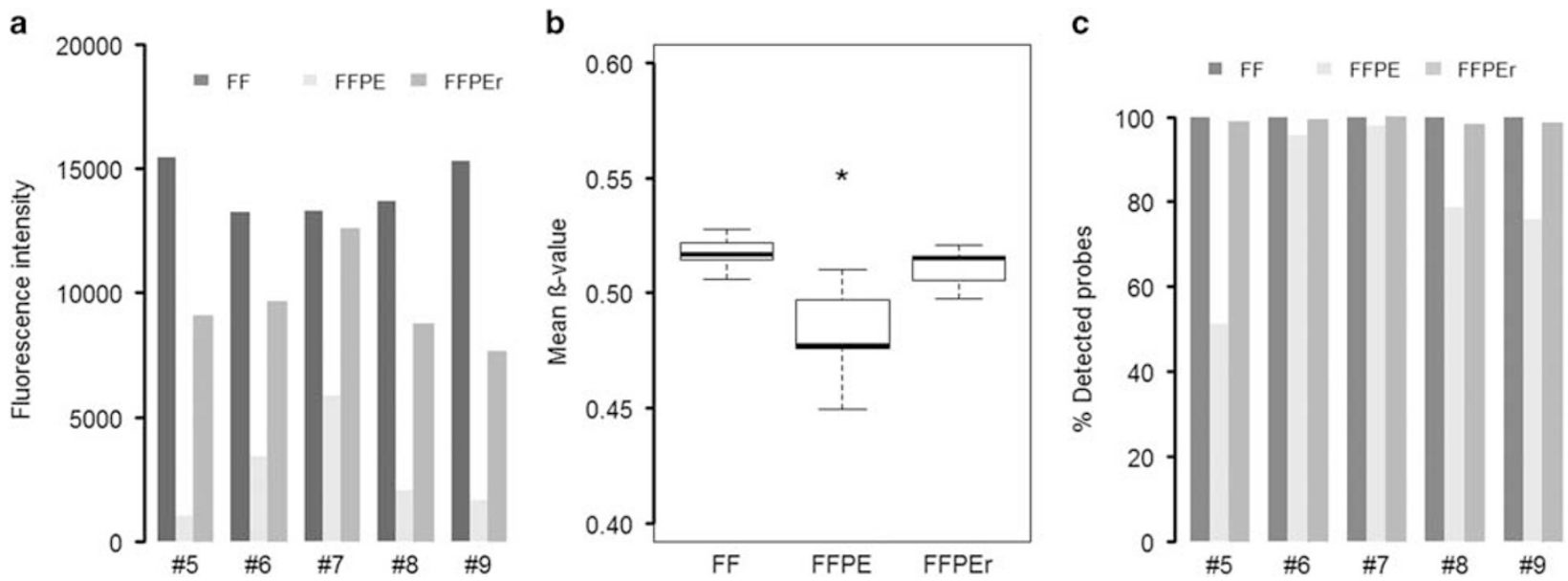

Figure 2 Fluorescence signal detection among DNA from different sample types. (a) Combined red and green signal intensity for five matched colon FF, FFPE and FFPEr samples shows improved probe binding of FFPE samples after DNA restoration. (b) Mean $\beta$-values of different matched FF, FFPE and FFPEr samples shows a significant $(* P=0.02)$ bias of FFPE samples towards unmethylated results. This bias no longer occurs in FFPEr samples. (c) Probe detection rate, defined as the percentage of the 485550 probes for each sample that yields a signal discernible from background with a $P$-value $<0.01$, for five matched FF, FFPE and FFPEr samples from the normal colon.

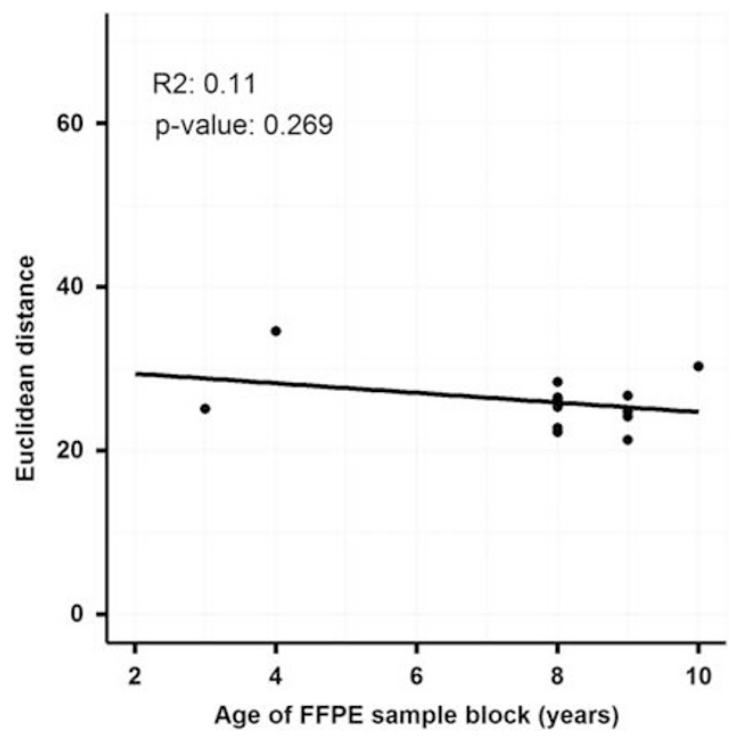

Figure 3 Euclidian distance plot with respect to sample archival age of FFPE tissues. Technical replicates of FFPE samples $(n=13)$ of varying age that have been used for $\mathrm{HM}-450 \mathrm{~K}$ analysis after DNA restoration show no significant association of assay performance with archival age of the sample.

\#9), show a lower reproducibility, especially when FFPEderived DNA is not restored prior to analysis. At a threshold of 0.2 absolute $\beta$-value difference, $5.7 \%$ of FFPEr-derived probes were discordantly methylated compared with their matched FF-derived counterparts, in contrast to $24.6 \%$ for FFPE- and FF-matched samples. In addition, non-restored FFPE samples show a clear lack of robustness, exhibiting a broad range of discordant methylation readout between samples (Figure 5b).

\section{Validation of the Discovery Potential of HM-450K Assays on Archival FFPEr-DNA}

In order to technically validate the discovery potential of the HM-450K assay when FFPEr-derived DNA is applied, we selected differentially methylated areas between colon mucosa (samples \#1 to \#9) and breast tumour samples (samples \#10 to \#20) according to HM-450K assay results. Nine genes contained areas that met the criteria as described in the Materials and Methods section. For five of these genes, oligonucleotide primers for nested MSP were designed and validated. The selected genes were NEU1 (sialidase 1), VWCE (von Willebrand factor C and EGF domains), GFI1 (growth factor independent 1 transcription repressor), MX2 (MX dynamin-like GTPase 2) and CMTM2 (CKLF-like MARVEL transmembrane domain containing 2).

First, we assessed the methylation status of the HM-450K assay samples using nested multiplex MSP. For four out of the five genes, MSP results confirmed the methylation status determined by the HM-450K assays (Figure 6a). Next, we expanded the initial cohort with 12 additional breast tumour samples and 12 additional colon mucosa samples. In this expanded cohort, again MSP results for the same four out of the five genes confirmed the results obtained from HM-450K assays. As shown in Figure 6a, GFI1, MX2 and VWCE, which were determined to be methylated in the breast but not in the normal colon based on $\mathrm{HM}-450 \mathrm{~K}$ results, were indeed methylated in 100, 100 and $91 \%$ of the breast tumour samples according to MSP, respectively, but not in any of the normal colon samples. The opposite was expected for NEU1, which was found to be methylated by MSP in 13\% of the breast tumour samples and in $100 \%$ of the normal colon samples. 


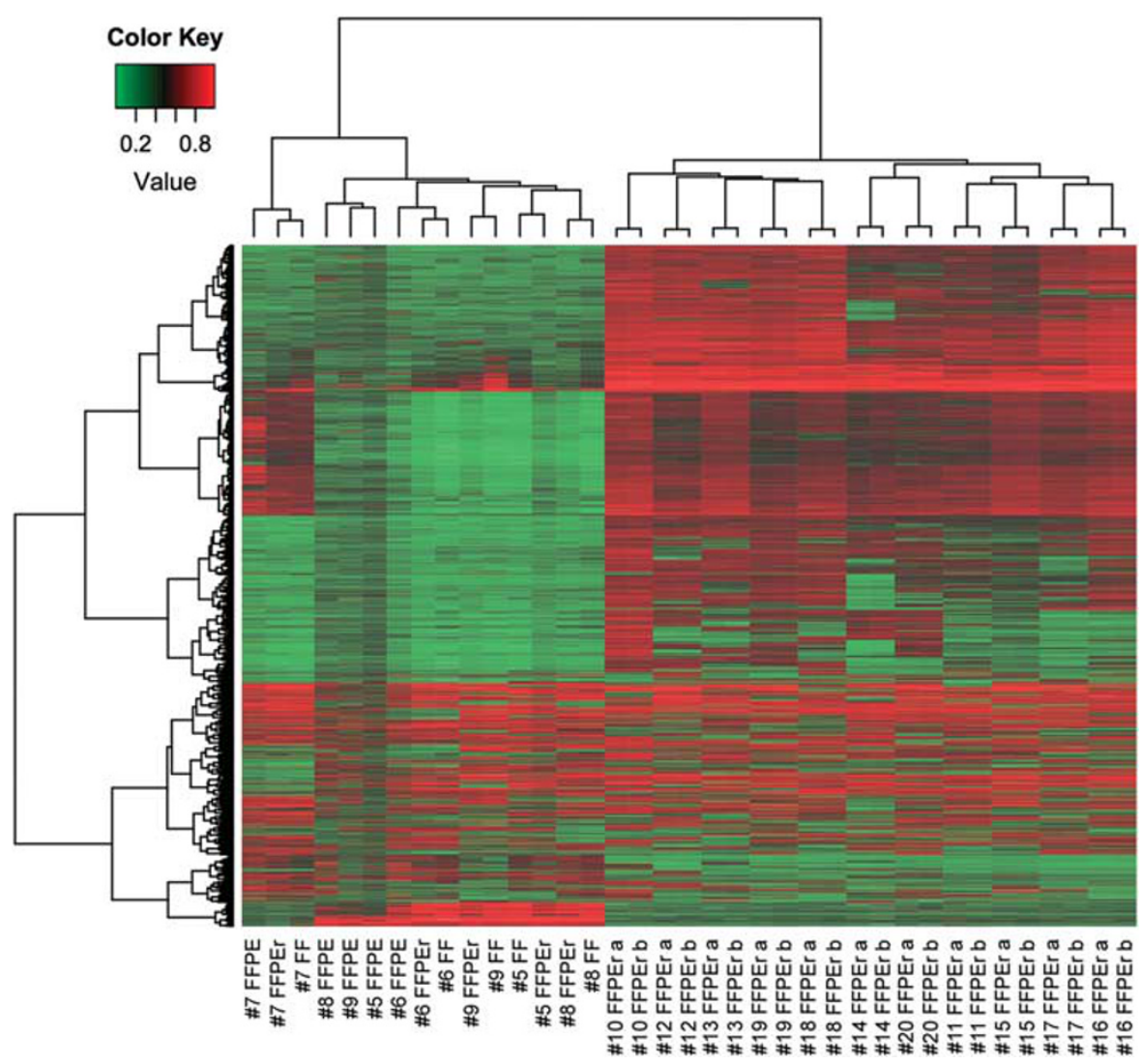

Figure 4 Unsupervised hierarchical clustering analysis. Unsupervised hierarchical clustering of the five matched FF, FFPE and FFPEr normal colon samples along with 11 breast tumour technical duplicates shows correct clustering of all duplicates and matched FF and FFPEr samples but not FFPE unrestored samples.

a

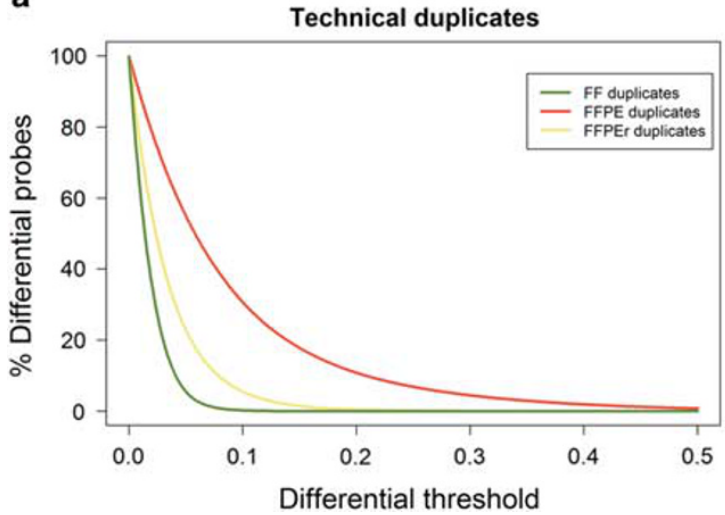

b

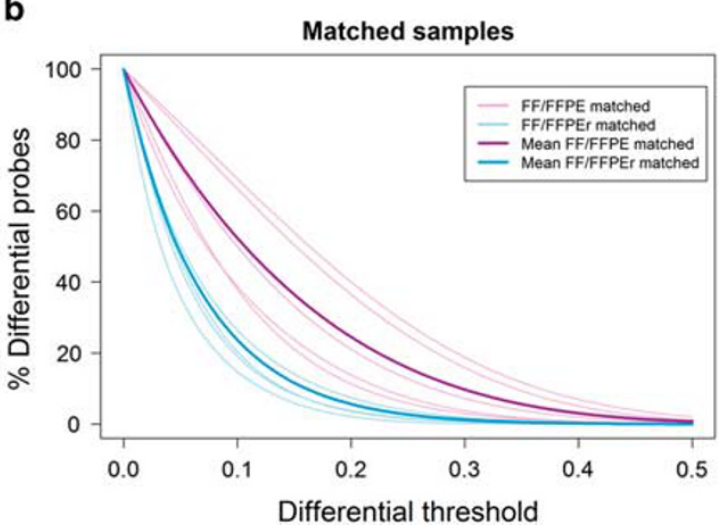

Figure 5 Percentage of CpG probes showing differential results set against $\beta$-value threshold, defined as absolute difference in $\beta$-value. (a) FFPEr technical duplicates show slightly more discordant CGs compared with FF technical duplicates. At a $\beta$-value threshold of 0.2 , there is no difference in reproducibility between FF- and FFPEr-derived results. Unrestored FFPE results show a high percentage of dissimilarity between duplicates. (b) When compared with FFPE results, FFPEr results show far better concordance with matched FF samples. Non-restored FFPE samples also show lack of robustness.

Discordantly, one gene (CMTM2) showed methylation in all the breast cancer and normal colon samples by MSP, although HM-450K results determined it to be unmethylated in the colon samples (Figure 6b).

\section{DISCUSSION}

Although archived FFPE tissue represents a rich and easy accessible source for molecular disease research, in the past years only two studies have used FFPE tissue-derived DNA on 
a

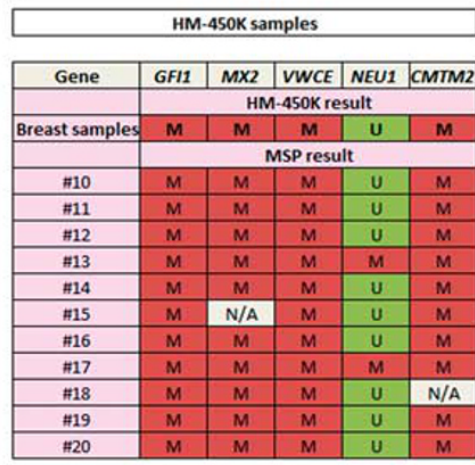

\begin{tabular}{|c|c|c|c|c|c|}
\hline Gene & GF11 & MX2 & VWCE & NEU1 & CMTM2 \\
\hline & \multicolumn{6}{|c|}{ HM-450K result } \\
\hline Colon samples & $U$ & U & U & M & U \\
\hline & \multicolumn{7}{|c|}{ MSP result } \\
\hline$\# 5$ & $U$ & $U$ & $U$ & $M$ & $M$ \\
\hline$\# 6$ & $U$ & $U$ & $U$ & $M$ & $M$ \\
\hline$\# 7$ & $U$ & $U$ & $U$ & $M$ & $M$ \\
\hline$\# 8$ & $U$ & $U$ & $U$ & $M$ & $M$ \\
\hline$\#$ & $U$ & $U$ & $U$ & $M$ & $M$ \\
\hline
\end{tabular}

b
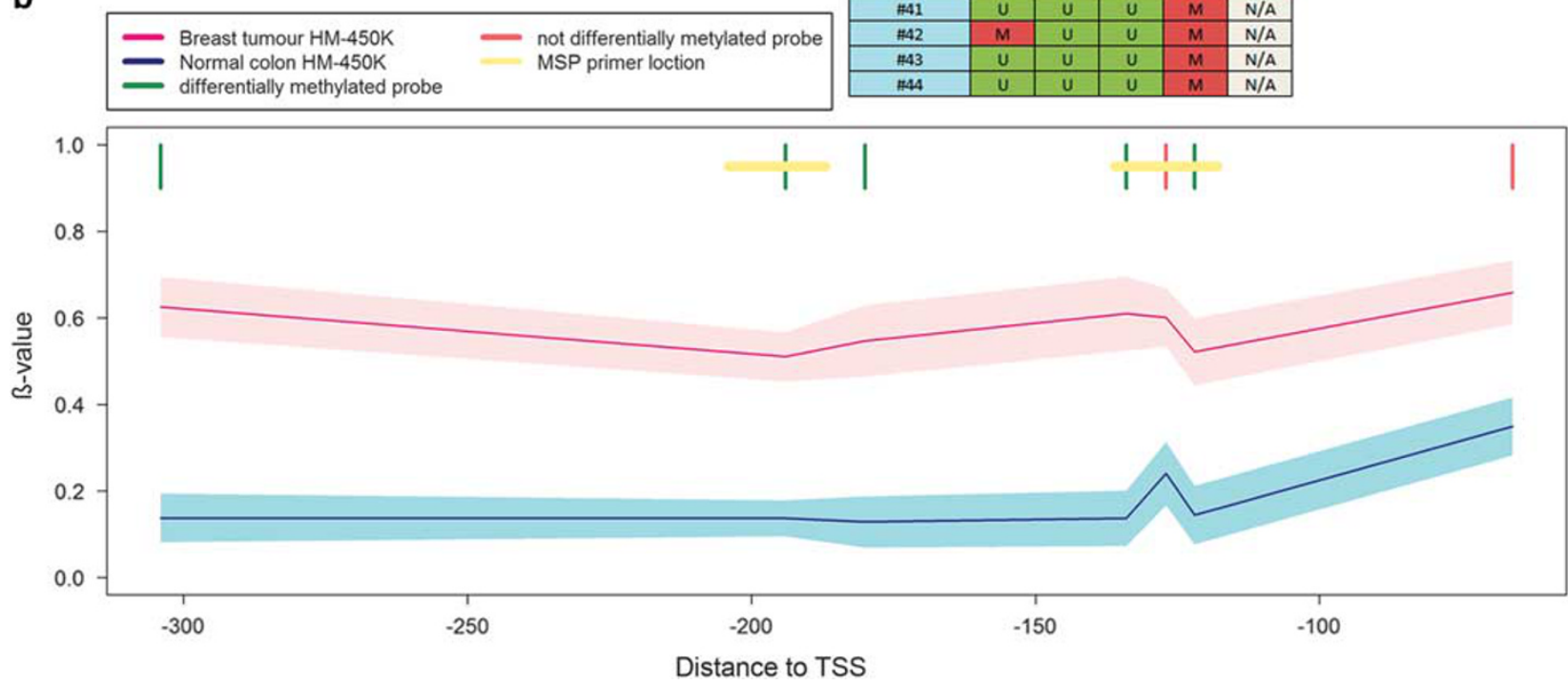

Figure 6 Nested MSP validation of differentially methylated genes. (a) MSP results of the HM-450K assayed breast tumour samples and normal colon samples as well as 24 additional breast tumour and normal colon samples. GFI1, MX2, VWCE and NEU1 reflect expected results based on HM-450K analysis. CMTM2 shows methylation in all samples, in contrast to expectations based on HM-450K results. (b) Location of nested multiplex MSP discriminatory primers in relation to HM-450K array probes for the CMTM2 area of interest in the colon samples shows one probe with a high $\beta$-value among probes with a low $\beta$-value possibly explaining failure of the validation of this gene with nested multiplex MSP. NA, not available (MSP failed); TSS, transcription start site.

Infinium HM-450K arrays to elucidate epigenomic alterations in cancer diseases. ${ }^{28,29}$ In comparison to the well-established use of HM-450K assays on FF tissues and cell lines, the application of FFPE-derived DNA in HM-450K assays may be hindered due to a lack of sufficient evidence proving the feasibility and reproducibility of this approach. Few recent studies describing the performance of HM-450K assays on FFPE tissue have shown a good concordance between matched FF and FFPE tissue when applying the Illumina
DNA restoration procedure on DNA from fixed tissue. ${ }^{19,20,30}$ However, only two of these studies analysed this concordance in a real-world scenario, ie, using long-time stored, archival tissues. Neither study has evaluated the reproducibility of results using both technical duplicates and matched FF/FFPE samples. Most importantly, these studies also did not test the potential of FFPEr-derived HM-450K results to identify differentially methylated genes in a second validation set of samples and confirm these by an independent technique, 
which is a prerequisite to successfully apply HM-450K assays for biomarker discovery research. ${ }^{19,20,30}$ We picked up on this lack of evidence and elaborated on the reproducibility of HM-450K with restored FFPE tissue-derived DNA by both analysing the concordance between technical duplicates and between matched FF and FFPEr biological replicates and influence of sample age on result reproducibility. In addition, we tested the potential of this approach to identify differentially methylated genes by a highly sensitive validation assay, i.e., nested multiplex MSP. For this study, two HM-450K subsets were available, one consisting of normal colonic mucosa samples that were processed first to assess improvement of assay quality after restoration of FFPE DNA and to investigate the influence of lower DNA input amounts. Based on these results, a second subset of breast tumour sample duplicates was processed to validate the findings of the first subset and to assess whether differentially methylated genes between the two subsets could be validated by MSP. Although from a biological point-of-view comparing normal colon mucosa to breast tumour tissue is of limited interest, this comparison provided us with information as to how the HM-450K assay performs as a discovery assay to select candidate differentially methylated genes.

Comparison of FF technical duplicates with FFPE and FFPEr technical duplicates showed a significant improvement of concordance between FFPE-derived duplicates after DNA restoration. As expected, FF-derived results obtained the highest concordance. Our results also demonstrate a good correlation between matched FF and FFPEr-derived results. In addition, the number of undetected probes per sample owing to weak signal intensity was low with FFPEr-derived DNA and comparable to FF samples. These findings are consistent with previously published findings from two other groups. ${ }^{19,20}$

To rule out tissue-type bias introduced by the use of FFPEr-derived DNA, we compared array signal intensities and $\beta$-values between matched samples. This showed no such bias, proving that use of either the FF or FFPEr protocol does not influence final results. In addition, analysis of discordant CG probes between technical and biological duplicates showed that, at a $\beta$-value cutoff of 0.3 , both FF and FFPEr samples yielded the same results. These analyses indicate that, using the Infinium DNA restoration protocol, the HM-450K assay can be used successfully with FFPE tissue-derived DNA. This conclusion is further substantiated by the unsupervised hierarchical clustering of the HM-450K assay results, which clustered all technical duplicates and matched samples correctly together, indicating high assay reproducibility.

Analysis of concordance between duplicates in relation to sample age did not yield any significant deterioration in archived samples up to 10 years of age. This may, however, be influenced by not including samples of low DNA integrity in the analysis, thus favouring high-quality results. As an additional novel finding, we have shown that reliable results can be achieved using a DNA input of only $100 \mathrm{ng}$, facilitating the analysis of smaller tissue samples, such as biopsies, as well.

In summary, our results prove a good correlation between FF and FFPEr samples on HM-450K assays and a high reproducibility using FFPEr-derived DNA.

We next verified whether FFPE-derived DNA analysed on HM-450K can be used as a discovery assay by selecting five genes that were differentially methylated between 9 normal colon and 11 breast cancer samples, according to FFPErderived $\mathrm{HM}-450 \mathrm{~K}$ assay results. We validated these genes using nested multiplex MSP, a highly sensitive PCR-based single gene methylation assay. ${ }^{31}$ Genes included those that have been previously described as methylation targets $\left(G I F 1^{32}\right.$ and $C M T M 2^{33}$ ), as well as genes that, to our knowledge, have previously not been reported as methylated (MX2, VWCE and NEU1). For four out of the five genes, differences in methylation found by the HM-450K assay could be confirmed using nested multiplex MSP. For one of the five selected genes (CMTM2), MSP results showed methylation in all samples, in contrast to the expected differentiation between the colon and breast tumour samples. Re-examination of the Infinium results of the CMTM2 area of interest showed one methylated probe among otherwise unmethylated probes in all the colon samples. This probe measures the methylation of a $\mathrm{CpG}$ located in the reverse primer used to assess CMTM2 methylation status by nested MSP, which likely accounts for the unexpected results for this gene. These results could be reproduced in an independent sample set. These data demonstrate that HM-450K assay discovery results can be validated using MSP. However, great care should be taken in MSP primer design as HM-450K assay methylation status may vary between neighbouring CpGs, whereas robust MSP results rely on a more homogeneous status of $\mathrm{CpGs}$ contained in the primer hybridisation sites, especially those towards the $3^{\prime}$ end of the primers.

In conclusion, our results clearly show that FFPE tissuederived DNA, after restoration using the Illumina Restoration Kit, can be a reliable source for DNA-methylome studies using the HM-450K assay. Along with results previously published, these findings pave the road for DNA-methylome discovery studies on archival tissue from cancer and other diseases. Supplementary Information accompanies the paper on the Laboratory
Investigation website (http://www.laboratoryinvestigation.org)

\section{ACKNOWLEDGMENTS}

The expert technical help from Kathleen Daenen (Department of Pathology, Maastricht University Medical (entre) is greatly appreciated. This study was supported by a research grant from AstraZeneca Netherlands (transDATA study; BOOG 2006-01, D5392-NL-003 - Sub study) to VCG Tjan-Heijnen and J Veeck.

\section{DISCLOSURE/CONFLICT OF INTEREST}

The authors declare no conflict of interest. 
1. Jones PA, Baylin SB. The fundamental role of epigenetic events in cancer. Nat Rev Genet 2002;3:415-428.

2. Petronis A. Epigenetics as a unifying principle in the aetiology of complex traits and diseases. Nature 2010;465:721-727.

3. Ebert MP, Tanzer M, Balluff B et al. TFAP2E-DKK4 and chemoresistance in colorectal cancer. N Engl J Med 2012;366:44-53.

4. Hegi ME, Diserens AC, Gorlia $T$ et al. MGMT gene silencing and benefit from temozolomide in glioblastoma. N Engl J Med 2005;352: 997-1003.

5. Moutinho C, Martinez-Cardus A, Santos C et al. Epigenetic inactivation of the BRCA1 interactor SRBC and resistance to oxaliplatin in colorectal cancer. J Natl Cancer Inst 2014;106:322.

6. Veeck J, Ropero S, Setien F et al. BRCA1 CpG island hypermethylation predicts sensitivity to poly(adenosine diphosphate)-ribose polymerase inhibitors. J Clin Oncol 2010;28:e563-e564.

7. van Vlodrop IJ, Niessen HE, Derks $\mathrm{S}$ et al. Analysis of promoter $\mathrm{CpG}$ island hypermethylation in cancer: location, location, location! Clin Cancer Res 2011;17:4225-4231.

8. Michels KB, Binder AM, Dedeurwaerder S et al. Recommendations for the design and analysis of epigenome-wide association studies. Nat Methods 2013;10:949-955.

9. Adusumalli S, Mohd Omar MF, Soong R et al. Methodological aspects of whole-genome bisulfite sequencing analysis. Brief Bioinform; advance online publication, 27 May 2014; pii: bbu016: doi:10.1093/bib/bbu016.

10. Berman BP, Weisenberger DJ, Aman JF et al. Regions of focal DNA hypermethylation and long-range hypomethylation in colorectal cancer coincide with nuclear lamina-associated domains. Nat Genet 2012;44:40-46.

11. Bibikova M, Le J, Barnes B et al. Genome-wide DNA methylation profiling using Infinium(R) assay. Epigenomics 2009;1:177-200.

12. Sandoval J, Heyn $\mathrm{H}$, Moran $\mathrm{S}$ et al. Validation of a DNA methylation microarray for 450,000 CpG sites in the human genome. Epigenetics 2011;6:692-702.

13. Dedeurwaerder S, Defrance $M$, Calonne $E$ et al. Evaluation of the Infinium Methylation 450K technology. Epigenomics 2011;3:771-784.

14. Bibikova M, Barnes $B$, Tsan C et al. High density DNA methylation array with single CpG site resolution. Genomics 2011;98:288-295.

15. Srinivasan $M$, Sedmak $D$, Jewell $S$. Effect of fixatives and tissue processing on the content and integrity of nucleic acids. Am J Pathol 2002;161:1961-1971.

16. Thirlwell C, Eymard M, Feber A et al. Genome-wide DNA methylation analysis of archival formalin-fixed paraffin-embedded tissue using the Illumina Infinium HumanMethylation27 BeadChip. Methods 2010;52: 248-254.

17. Jasmine F, Rahaman R, Roy $\mathrm{S}$ et al. Interpretation of genome-wide infinium methylation data from ligated DNA in formalin-fixed, paraffinembedded paired tumor and normal tissue. BMC Res Notes 2012; $5: 117$
18. Thirlwell C, Feber A, Lechner $M$ et al. Comments on: Interpretation of genome-wide infinium methylation data from ligated DNA in formalinfixed paraffin-embedded paired tumor and normal tissue. BMC Res Notes 2012;5:631.

19. Dumenil TD, Wockner LF, Bettington $M$ et al. Genome-wide DNA methylation analysis of formalin-fixed paraffin embedded colorectal cancer tissue. Genes Chromosomes Cancer 2014;53:537-548.

20. Moran S, Vizoso M, Martinez-Cardus A et al. Validation of DNA methylation profiling in formalin-fixed paraffin-embedded samples using the Infinium HumanMethylation450 Microarray. Epigenetics 2014;9:6.

21. Siegel $E M$, Berglund $A E$, Riggs $B M$ et al. Expanding epigenomics to archived FFPE tissues: an evaluation of DNA repair methodologies. Cancer Epidemiol Biomarkers Prev 2014;23:2622-2631.

22. Burdorf $A$, Hollema $H$, van Leeuwen $E$ et al. Human tissue and medical research: Code of conduct for responsible use. Stichting FMWV Rotterdam, 2011.

23. Federation of Biomedical Scientific Societies. Code of conduct for Health Research. Stichting FMWV, Rotterdam, 2011.

24. Alders M, Bliek J, vd Lip K et al. Determination of KCNQ1OT1 and H19 methylation levels in BWS and SRS patients using methylationsensitive high-resolution melting analysis. Eur J Hum Genet 2009;17: 467-473.

25. Derks S, Lentjes MH, Hellebrekers DM et al. Methylation-specific PCR unraveled. Cell Oncol 2004;26:291-299.

26. Herman JG, Graff JR, Myohanen S et al. Methylation-specific PCR: a novel PCR assay for methylation status of CpG islands. Proc Natl Acad Sci USA 1996;93:9821-9826.

27. van Engeland $M$, Roemen GM, Brink $M$ et al. K-ras mutations and RASSF1A promoter methylation in colorectal cancer. Oncogene 2002;21:3792-3795

28. Killian JK, Kim SY, Miettinen M et al. Succinate dehydrogenase mutation underlies global epigenomic divergence in gastrointestinal stromal tumor. Cancer Discov 2013;3:648-657.

29. Lechner M, Fenton T, West J et al. Identification and functional validation of HPV-mediated hypermethylation in head and neck squamous cell carcinoma. Genome Med 2013;5:15.

30. American Cancer society, Global Cancer Facts \& Figures 2nd (edn). American Cancer Society: Atlanta, GA, USA, 2011.

31. Divine KK, Liechty KC, Crume KC et al. Nested multigene MSP/DHPLC method for analyzing promoter hypermethylation status in clinica samples. Biotechniques 2006:40:40-48.

32. Elliott HR, Tillin T, McArdle WL et al. Differences in smoking associated DNA methylation patterns in South Asians and Europeans. Clin Epigenetics 2014;6:4.

33. Fang WJ, Zheng $\mathrm{Y}, \mathrm{Wu} \mathrm{LM}$ et al. Genome-wide analysis of aberrant DNA methylation for identification of potential biomarkers in colorectal cancer patients. Asian Pac J Cancer Prev 2012;13:1917-1921. 